\title{
Leakage Mechanism of Soft Plunger Pumps
}

\author{
Chunying $\mathrm{Hu}^{1,2}$, Guoyou Han ${ }^{1, *}$, Weijian Chen ${ }^{1}$, Weidong $\mathrm{Ye}^{1}$, and Daoquan Han ${ }^{1,3}$ \\ ${ }^{1}$ School of Mechanical Science and Engineering, Northeast Petroleum University, Daqing 163318, China \\ ${ }^{2}$ College of Engineering, Heilongjiang Bayi Agricultural University, Daqing 163319, China \\ ${ }^{3}$ Khartoum Refinery Co. Ltd, Khartoum North 12211, Sudan
}

Received 7 October 2018; Accepted 19 December 2018

\begin{abstract}
As a new type of petroleum extraction device, soft plunger pumps can effectively avoid the stuck problem of pump and realize the lift of underground petroleum. Meanwhile, soft plunger pumps have strong adaptability to wells using Alkaline surfactant polymer flooding. Non-metallic sealing rings have experienced frequent leakage under the influence of pressure difference due to the increase in the extraction difficulty and the submergence depth, thereby reducing their pumping efficiency eventually. Therefore, studies on the leakage of soft plunger pumps are crucial for the calculation of plunger velocity and the improvement of pumping efficiency. A model for multistage soft plunger pumps was proposed in this study to reveal the relationship between the leakage amount and the operational and structural parameters of soft plunger pumps. The finite element method was used to determine the length and thickness of the sealing ring in the paper, thereby establishing a physical model. The influence factors on the leakage amount, namely, pressure difference, velocity, medium viscosity and clearance, were analyzed on the basis of fluid dynamics and the theorem of momentum. The influences were also explored by using theoretical analysis and empirical test. In addition, a prediction model was constructed on the basis of the operational characteristics of soft plunger pumps to verify the correctness of the leakage mechanism. Results show that the leakage amount of soft plunger pumps increases with the pressure difference under the premise of constant medium viscosity, velocity and clearance, in which the leakage amount in high-viscosity medium is smaller than that in low-viscosity medium. In the pressure difference, medium viscosity and clearance remain unchanged and the volumetric efficiency of soft plunger pumps could be considerably enhanced by increasing the velocity. Given that large clearances are found to intensify pump leakage, maintaining the clearance in the range of 0.2 to $0.5 \mathrm{~mm}$ is recommended. The study provides a theoretical basis for correctly improving the pumping efficiency of soft plunger pumps.
\end{abstract}

Keywords: Soft plunger, Pump, Leakage amount

\section{Introduction}

Current petroleum extraction devices mainly include plunger, screw, electric submersible, hydraulic and gas lift pumps. Among these pumps, plunger pumps, which achieve outstanding economic and social benefits, serve as the earliest and the most extensively used petroleum extraction device. Plunger pumps have been applied extensively to petroleum extraction from ordinary, deep, ultra-deep, and heavy oil wells due to their advantages of simple structure, convenient operation, high reliability, strong adaptability, excellent lifting performance and protection for oil pipes. The demand for plunger pumps has shown a trend of rapid growth, and studies on related techniques have rapidly progressed. Meanwhile, a growing number of units have participated in the study, design, production, and application of plunger pumps. Plunger pumps can be further divided into hard and soft plunger pumps based on sealing materials. Given that hard plunger pumps are easily stuck, the China University of Petroleum has presented a soft plunger pump that relies on the hydraulic pressure to realize sealing.

Petroleum extraction is currently in the second or third stage when the petroleum reserves are reduced and the

*E-mail address: hchunying@163.com

ISSN: $1791-2377$ @ 2018 Eastern Macedonia and Thrace Institute of Technology. All rights reserved. doi:10.25103/jestr.116.10 extraction conditions are complicated. Petroleum reserves tend to contain some free gas. A common method to reduce the influence of free gas is to increase the submergence depth of pumps. The inlet pressure increases with the submergence depth. The rubber sealing rings experience an increase in elasticity under pressure, thereby causing fatigue failures (transiting from fatigue crack initiation to crack growth). Consequently, the pumps leak seriously and therefore fail. The leakage of pumps involves numerous factors, which not only increase the technical difficulty but also pose a tremendous challenge to the study on soft plunger pumps.

Therefore, numerous studies have been conducted on the materials of sealing rings and the leakage amount and lifting performance of soft plunger pumps [1-3]. Scholars have optimized the materials of sealing rings and revealed the relationship between leakage amount and lifting performance. However, few studies have been concerned on the influences of pressure difference, velocity, medium viscosity, and geometric parameters on leakage amount, thereby distancing the physical models and operational parameters of soft plunger pumps from reality. Existing studies cannot solve the problems of pumping efficiency and lifetime. Therefore, exploring the leakage mechanism, accurately predicting the leakage, analyzing basic factors that affect the leakage amount, and clarifying the coupling 
relationship between the operational parameters and physical models of soft plunger pumps in operation are urgent.

A model for multistage soft plunger pumps applicable to Alkaline surfactant polymer flooding was designed in this study to lower the pressure on single-stage plunger pumps, extend the service life of soft plunger pumps, and achieve uniform pressure at each stage. The proposed model was established by using the finite element method to analyze the leakage of soft plunger pumps under different pressure differences, velocities, medium viscosities and clearances. This study aims to predict the coupling relationship between the operational parameters and physical models of soft plunger pumps accurately, thereby providing references for the optimization of related parameters.

\section{State of the Art}

Soft plunger pumps have been developed in many countries and applied to on-site petroleum extraction. Statistics show that the service life of soft plunger pumps is $55 \%-60 \%$ longer than that of ordinary pumps [4]. Thus far, scholars have conducted numerous studies on soft plunger pumps.

Cui et al. [5-6] studied the transmission laws of the pressure of soft plunger pumps and matched the shape of decompression tanks with the angle of pressure transmission holes to reduce the wear of soft sealing cups, thereby realizing the uniform distribution of pressure and achieving the equivalent life design of sealing cups. However, no costeffective wear-resistant materials were selected to solve the valve leakage caused by erosion. Thus, enhancing the hightemperature and aging resistance of sealing cups and prolonging the service life of soft plungers are urgent. Wang Yanli et al. [7-8] verified the strong sealability of flexible plunger and retrievable standing valve pumps by performing wear, pressure, and leakage tests in Daqing Oilfield. The leakage amount was found to be close to zero, and the single-well pumping efficiency was improved by $20 \%$ on average. Nevertheless, the proposed pumps were not applicable to sand-producing wells. $\mathrm{Li}$ and $\mathrm{Sun} \mathrm{[9-10]}$ conducted experimental comparison to analyze the sensitivity of conventional pumps and pumps with sectionalized soft plungers to sands suspended in the strings. The results showed that pumps with sectionalized soft plungers operated smoothly and effectively avoided stuck pumps caused by scales or sands under sandy conditions. Given that the soft plungers were installed every $300 \mathrm{~m}$ and the pumping efficiency was below $1000 \mathrm{~m}$, the application range of such pumps was restricted. Tripp [11] and Zhu WB [12] developed an ant-struck pump with combined plungers to address the deficiencies of traditional pumps. Although the pump detection period was effectively shortened during the test in the Third Oil Production Plant of Daqing Oilfield, implementing the design of combined plungers in the case of undersize diameters was difficult. Newton [13] and Randle [14] established a solid model of anti-stuck pumps based on the analysis of the structural characteristics of anti-stuck pumps in ASP flooding. The ANSYS software was adopted to simulate the sealing of anti-stuck pumps, and the multivariate orthogonal experimental design method was used to optimize the structural parameters. In addition, wear and leakage tests were performed for model verification. Given the complex conditions of wells using ASP flooding, wear failures were likely to occur, which indicated the necessity of further explorations. Metelkin et al. [15-17] explored the leakage amount of hydraulic self-sealing plunger pumps based on the fluid dynamics and revealed the superiority of these plunger pumps in leakage amount. When the clearance ranged between $0.1 \mathrm{~mm}$ and $0.15 \mathrm{~mm}$, the leakage amount of hydraulic self-sealing plunger pumps lifting polymer-containing viscoelastic fluids was considerably lower than that specified in API SPEC 11AXBIAX. Meanwhile, the leakage amount was lower than that of conventional plunger pumps under the premise of constant clearance. However, studies on the off-center wear of rods in polymer flooding wells are lacking. Li J. et al. [18] adopted the orthogonal design method to discuss the flexible pumping system of plunger pumps. The clearance was determined based on multiple optimization experiments, thereby solving the problems of rubber swelling and excessive pull-up load. Unfortunately, the sealing and expansion rings of such hydraulic self-sealing soft plunger pumps have poor wearability and short service life.

Overall, previous studies were mainly focused on the design and structure of soft plunger pumps. Few studies concerning the leakage characteristics, especially the influences of the structural and operational parameters of soft plunger pumps on the leakage amount, were available. Therefore, a physical model for soft plunger pumps was constructed by using stress analysis and computational fluid dynamics to study the clearance flows between the sealing ring and pump barrel. A mathematical model of the leakage amount was also established. The leakage characteristics of soft plunger pumps were analyzed under different pressure differences, velocities, viscosities and clearances, thereby providing a basis for the optimization and test study on soft plunger pumps.

The remainder of the study is organized as follows: First, a physical model for two-stage soft plunger pumps is established to study the clearance flows and explore the influence factors on the leakage amount. Second, the leakage characteristics of the proposed model are analyzed by using the finite element method, thereby obtaining leakage amount under different conditions. Finally, relevant conclusions are drawn.

\section{Methodology}

\subsection{Physical Model}

The structure of the two-stage soft plunger pumps is shown in Figure 1. During the upstroke, the sucker rod moves upward, and the conical section of the sucker rod gets stuck to the soft plunger to form a sealed space. The space below the plunger increases accordingly. Given the changes in pressure, the traveling valve moves upward, thereby opening the standing valve. Consequently, the fluid is sucked into the pump barrel, and the pumping process is completed. Meanwhile, the chuck of the sucker rod is separated from the upper end of the soft plunger during the upstroke. The fluid enters the hollow part of the plunger and flows through its radial groove to form lateral force on the sealing ring. Therefore, the sealing ring experiences elastic deformation and satisfies the requirements of pumping efficiency. Some of the fluid flows through the clearance of the first-stage plunger to enter the second-stage plunger. The pressure difference follows the declining laws, which indicates that the pressure on each stage of sealing elements is considerably smaller than that on single-stage soft plungers. In addition, the second-stage sealing ring can still play a role in sealing and pressure distribution despite the occurrence of leakage due to the wear and friction between the sealing ring 
and the pump barrel in the first-stage soft plunger. Therefore, two-stage soft plunger pumps achieve higher social benefits and application values than single-stage soft plunger pumps. During the downstroke, the sucker rod moves downward, the chuck contacts the upper end of the soft plunger, and the conical section separates itself from the lower end of the soft plunger. Given that the chuck of the sucker rod is uniformly perforated in the radial direction, the fluid in the pump barrel flows through the hollow part of the plunger and enters the chuck and then moves upward, thereby completing the oil draining process. The sealing ring restores its original shape due to the uniform pressure. Consequently, the downstroke resistance is reduced, and the lubrication between the friction pairs is ensured.

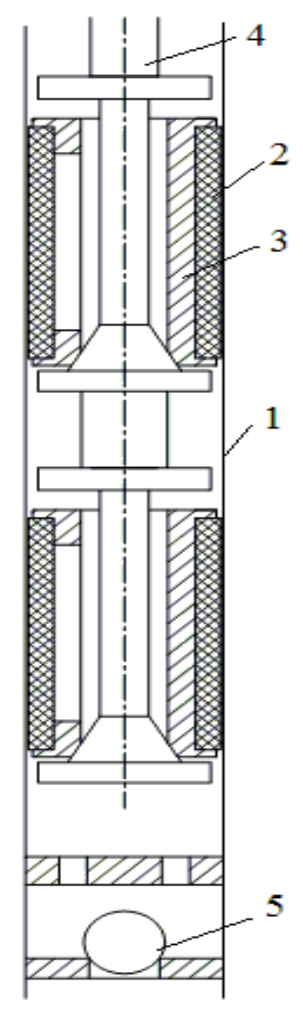

1 - Pump barrel; 2 - Sealing ring; 3 - Plunger; 4 - Sucker rod; 5 Traveling valve

Fig. 1. Structural Diagram of Two-Stage Soft Plunger Pumps

\subsection{Determination of Key Parameters}

The sealing ring (also known as the sleeve) is arranged in the sealing groove to form a clearance fit with the pump barrel. The sealing rings of soft plunger pumps are generally composed of Buna-N, PTEF, HMWPE, and PEEK, while the pump barrels usually comprise chrome-plated alloy steel. The sealing rings and the pump barrels jointly constitute friction pairs. Given that wear resistance is an important index, PEEK is eventually selected in this study after comparing the wear resistance of Buna-N, PTEF, HMWPE, and PEEK. The ANSYS software is used in calculating the contact stress to determine the length and thickness of the sealing ring. During the upstroke, the contact stress serves as a main factor that affects the leakage and lifting performance of soft plunger pumps. The contact stress and length differ among sealing rings. On the basis of the contact stress analysis of sealing rings of different lengths (Figure 2), the maximum contact stress, contact position, and effective contact length (contact length $>8 \mathrm{MPa}$ ) can be obtained. Stress comparison curves of sealing rings of different lengths are drawn to facilitate the analysis, as shown in Figure 3. From the figure, the maximum contact stress is 8.3 $\mathrm{MPa}$ and the effective contact length is $2 \mathrm{~mm}$ when the length of the sealing ring is $50 \mathrm{~mm}$. When the length of the sealing ring is $70 \mathrm{~mm}$, the maximum contact stress and effective contact length are $8.5 \mathrm{MPa}$ and $5.5 \mathrm{~mm}$ separately. When the length of the sealing ring is $60 \mathrm{~mm}$, the maximum contact stress and effective contact length are $8.4 \mathrm{MPa}$ and 4 $\mathrm{mm}$, respectively. Given that wear occurs easily in the case of high contact stress and large contact length, $60 \mathrm{~mm}$ is selected as the optimal length of sealing rings.

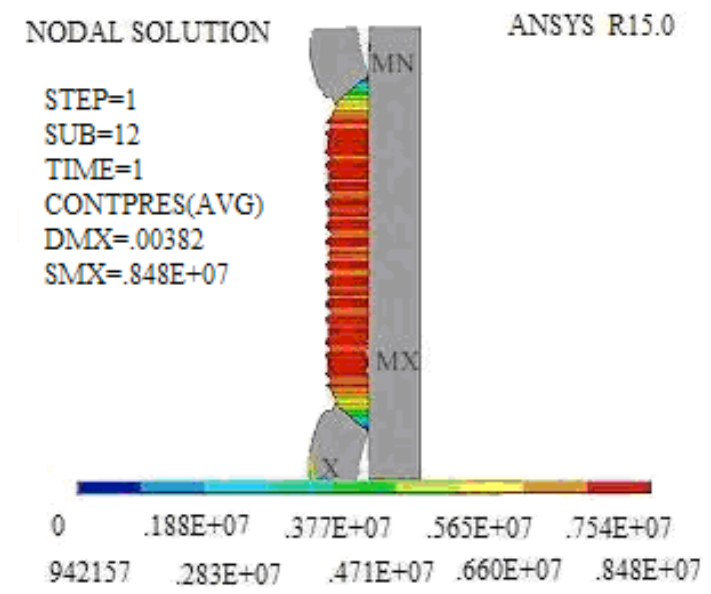

(a)

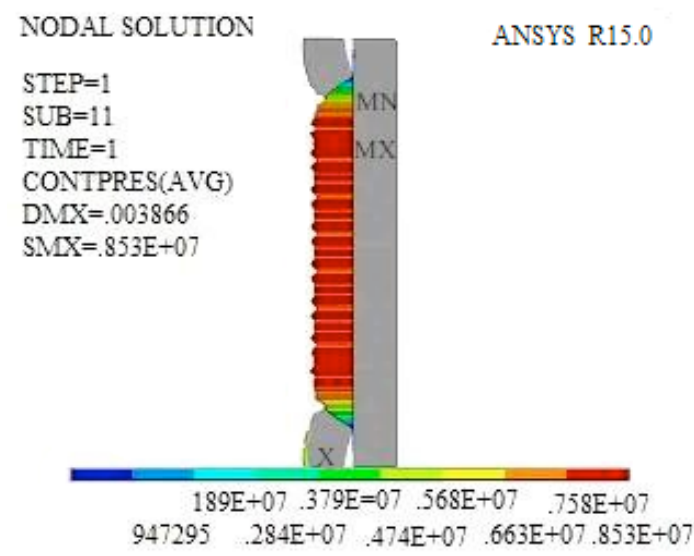

(b)

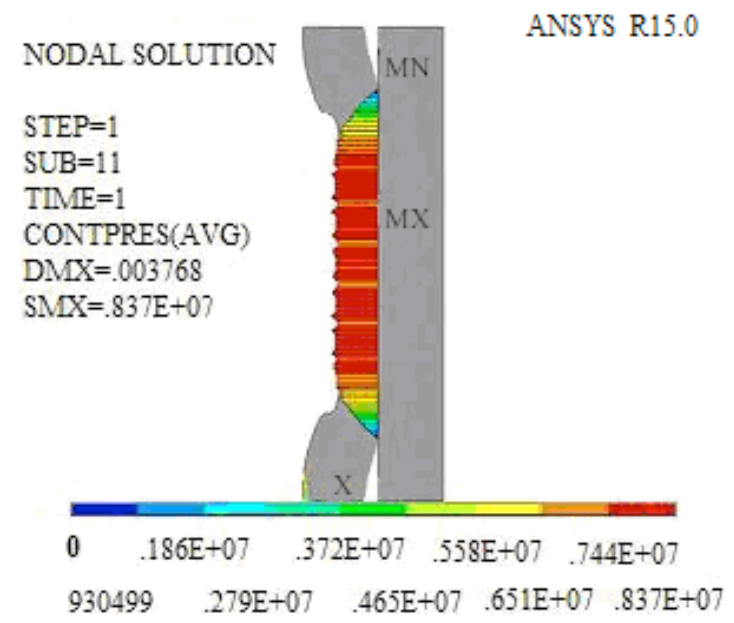

(c)

Fig. 2. Contact Stress of Sealing Rings with Different Lengths. (a) Length of Sealing Ring $=60 \mathrm{~mm}$. (b) Length of Sealing Ring $=70 \mathrm{~mm}$ (c) Length of Sealing Ring $=50 \mathrm{~mm}$ 


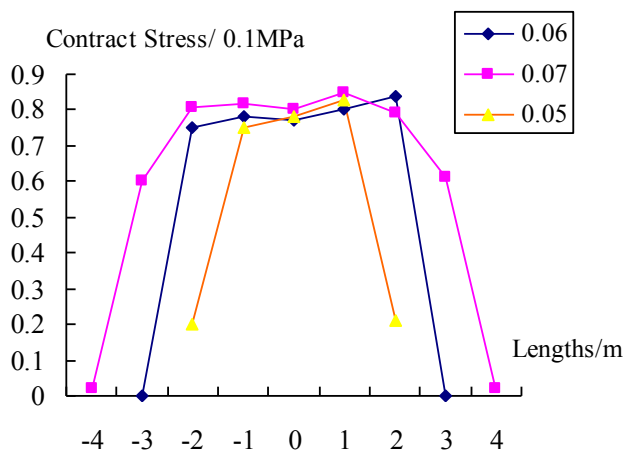

Fig. 3. Stress Comparison of Sealing Rings with Different Lengths

Similarly, stress comparison curves of sealing rings with different thicknesses are drawn, as shown in Figure 4. From the figure, the effective sealing area reaches its maximum value when the thickness is $6 \mathrm{~mm}$. However, the sealing ring undergoes considerable contact stress changes and large local deformations in that case. When the thickness is 7.5 $\mathrm{mm}$, the effective contact length is the shortest and the sealing effects are poor due to the large thickness. Although the sealing ring achieves moderate deformation and effective contact length in both cases (thickness $=6.5 \mathrm{~mm}$ and $7 \mathrm{~mm}$ ), $7 \mathrm{~mm}$ is selected as the optimal thickness for convenience.

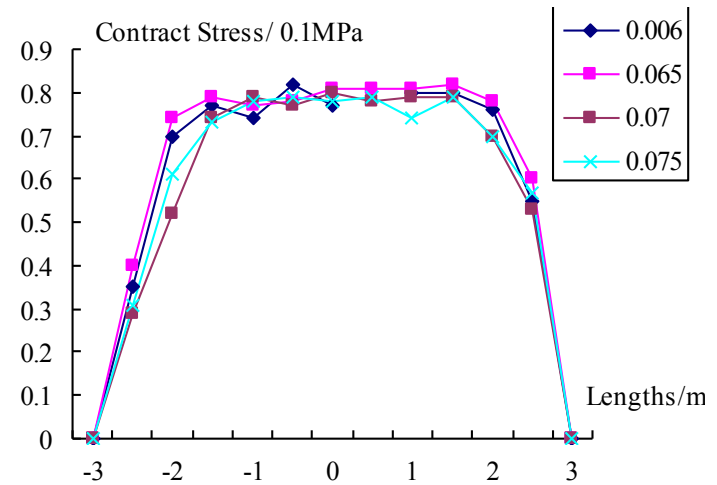

Fig. 4. Stress Comparison of Sealing Rings with Different Thicknesses

\subsection{Clearance Flow}

Clearance flows play an important role in elastohydrodynamic lubrication, thereby reducing the friction between the sealing ring and the pump barrel [1921]. The proposed model is simplified as a parallel plate model conforming to the $\mathrm{N}-\mathrm{S}$ equation or the Reynolds equation to study the annular clearance flows between the sealing ring and the pump barrel and analyze the characteristics of the clearance flows, as shown in Figure 5.

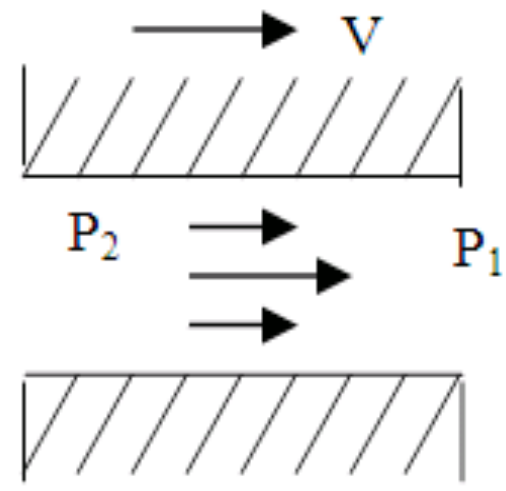

Fig. 5. Model of Equivalent Clearance Flows
As shown in Figure 5, the length of the parallel plates is 1; the height and width of the clearance between the two parallel plates are $\mathrm{h}$ and $\mathrm{b}$, respectively $(l>>h, b>>h)$. The liquid is incompressible, the mass force is neglected, and the fluid viscosity is kept constant. Fluid flows between the two parallel plates can be regarded as laminar flows. On the basis of the fluid dynamics and the theorem of momentum, the clearance flows can be expressed as:

$Q=\int_{u} d A=\int_{0}^{h} \mu b d z=-b \int_{0}^{h} \frac{1}{2 \mu} \frac{d p}{d x}(h-z) z d z$

where $\mu$ is the dynamic viscosity of the medium.

If the two parallel plates are relatively static, then only flows in the $x$ - and $y$-directions are considered when the height of the clearance is extremely small. Thus, the clearance flows can be obtained by:

$Q=-\frac{b}{2 \mu} \frac{d p}{d x} \int_{0}^{h}(h-z) z d z=-\frac{b}{12 \mu} \frac{d p}{d x} h^{3}$

The pressure distribution gradient can be expressed as follow:

$d p / d x=-12 \mu Q_{p} / b h^{3}=$ const

Equation (3) shows that the pressure is linear in the $\mathrm{x}$ direction, and the pressure difference is $\Delta p=p_{2}-p_{1}$ during pressure drops. On this basis, the pressure change ratio can be obtained based on the equation $d p / d x=\left(p_{2}-p_{1}\right) / l=\Delta p / l$. The flows are calculated as:

$Q=\frac{b h^{3} \Delta p}{12 \mu l}$

where $b$ is the width of the clearance, $h$ is the height of the clearance, $\Delta p$ is the inlet and outlet pressure difference, $\mu$ is the dynamic viscosity of the medium, and 1 is the total length of the clearance.

If the two parallel plates are relatively dynamic, then the clearance flows can be expressed as:

$Q=\int_{0}^{h} u b d z=b \int_{0}^{h}\left[-2 \frac{1}{2 \mu} \frac{d p}{d x}(h-z) z+v\left(\frac{h-z}{z}\right)\right] d z$

The following equation can be obtained by calculating the integral of Equation (5):

$Q=\frac{b h^{3} \Delta p}{12 \mu l}+\frac{b h}{2} v$

where $v$ is the relative velocity between the two parallel plates.

Two concentric cylindrical surfaces can form an annular clearance. By setting the width of the clearance as a constant $h$ and replacing $b$ in Equation (6) with the perimeter of the annular clearance $\pi d$, the clearance flows can be obtained as follows: 
$Q=\frac{\pi d h^{3} \Delta p}{12 \mu L}$

The two cylindrical surfaces are relatively rotated at a velocity $v$. In this case, the clearance flows can be expressed as:

$Q=\frac{\pi d h^{3} \Delta p}{12 \mu L}+\frac{\pi d h}{2} v$

The clearance flows between the sealing ring and pump barrel are the main manifestations of leakage. Equation (8), which serves as a mathematical model of the leakage amount, reveals the functional relationship between the leakage amount and the length, thickness, clearance, pressure difference, and velocity of the sealing ring. Equation (8) also provides a basis for the analysis of the relationship between the leakage amount and various influence factors.

\subsection{Experimental Verification}

Figure 6 shows a picture of the simulation experimental apparatus, including the self-made pumping unit, pump and accessories, such as sealing ring. During the experiment, the length and the number of strokes can be adjusted to meet the requirements of the stroke length, number and momentum. The downhole conditions of soft plunger pumps are simulated, thereby verifying the influences of velocity, medium viscosity, clearance, and pressure difference on the leakage amount.

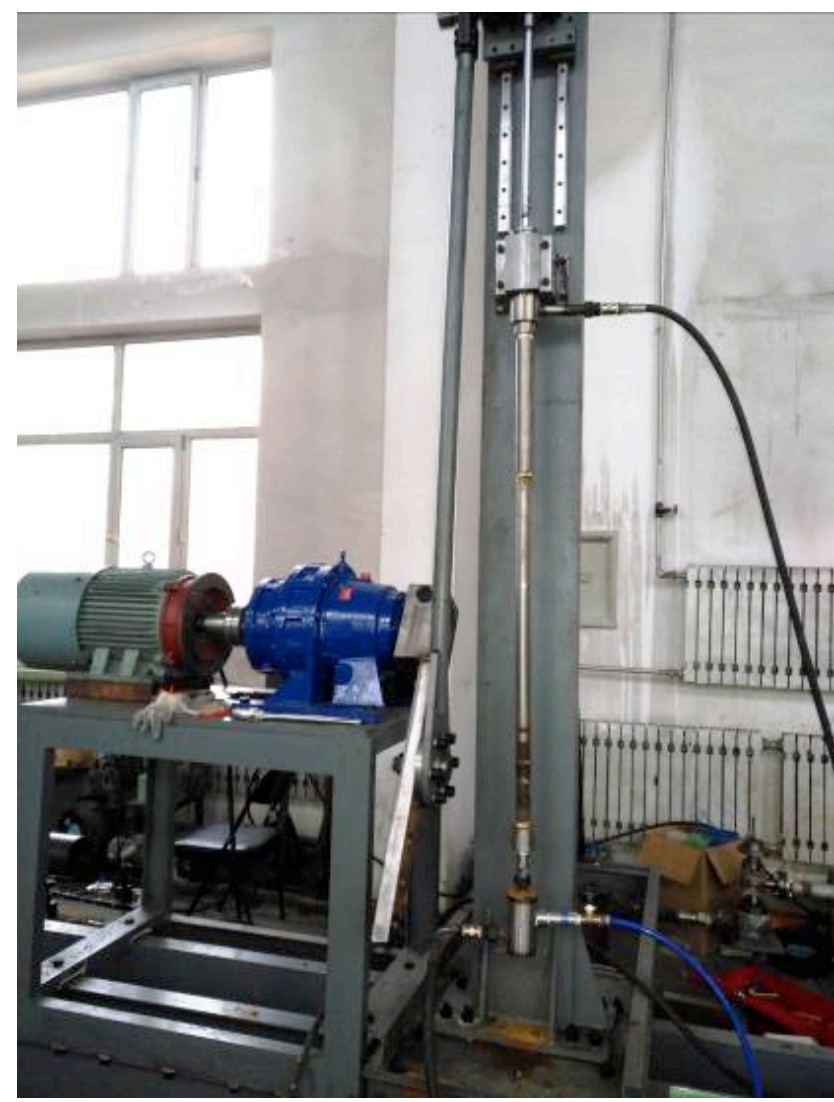

Fig. 6. Simulation Experimental Apparatus

\section{Result Analysis and Discussion}

On the basis of the calculation model in Section 3.3, the ANSYS software was used to analyze the leakage mechanism of multistage soft plunger pumps and explore the influences of pressure difference, medium viscosity, clearance, and velocity on leakage amount, thereby providing theoretical support for the experimental verification in Section 3.4.

4.1 Influence of Pressure Difference on Leakage Amount The influence of pressure difference on leakage amount was analyzed under the premise of constant medium viscosity (35 Cp), fluid density $(830 \mathrm{~kg} / \mathrm{m} 3)$, and velocity $(20 \mathrm{~L} / \mathrm{min})$. Figure 7 shows that the leakage amount under the pressure differences of $0.1,0.2,0.3,0.4$ and $0.5 \mathrm{MPa}$ are $0.05,0.12$, $0.19,0.27$ and $0.35 \mathrm{~kg} / \mathrm{s}$ respectively. The leakage amount increases by approximately $0.07 \mathrm{~kg} / \mathrm{s}$ for every $0.1 \mathrm{MPa}$ increase in the pressure difference. Thus, the leakage amount increases with the pressure difference. When the inlet and outlet pressure difference is fixed, the pressure difference can be uniformly distributed by changing the number of stages. Therefore, changes in pressure difference can be equivalent to the changes in the number of stages. When the pressure difference remains constant, the number of stages of soft plunger pumps can be appropriately increased to reduce the single-stage pressure difference, thereby effectively reducing the leakage amount.

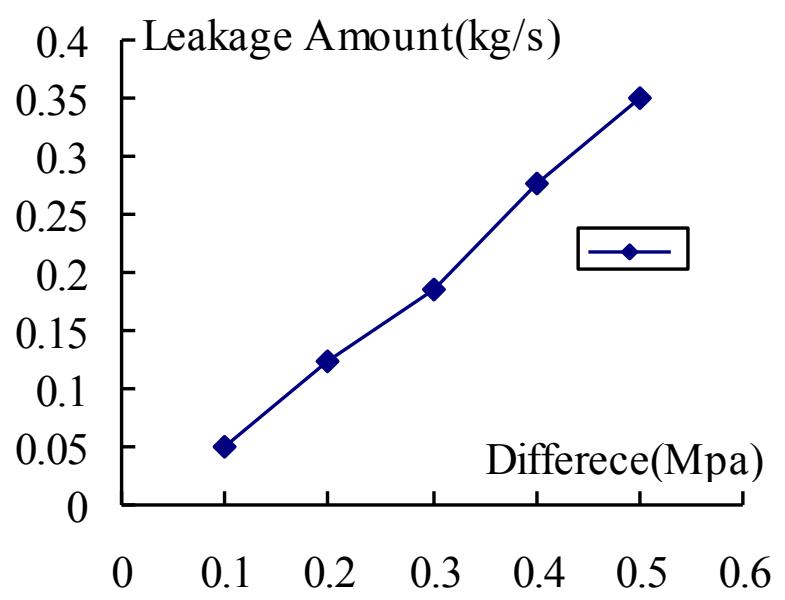

Fig. 7. Changes of Leakage Amount with Pressure Difference

4.2 Influence of Medium Viscosity on Leakage Amount Medium viscosity is an important factor that affects the leakage amount of soft plunger pumps. Figure 8 demonstrates the leakage amount of soft plunger pumps under different medium viscosities $(20,40,60,80,100,120$, 140 , and $160 \mathrm{cP})$.

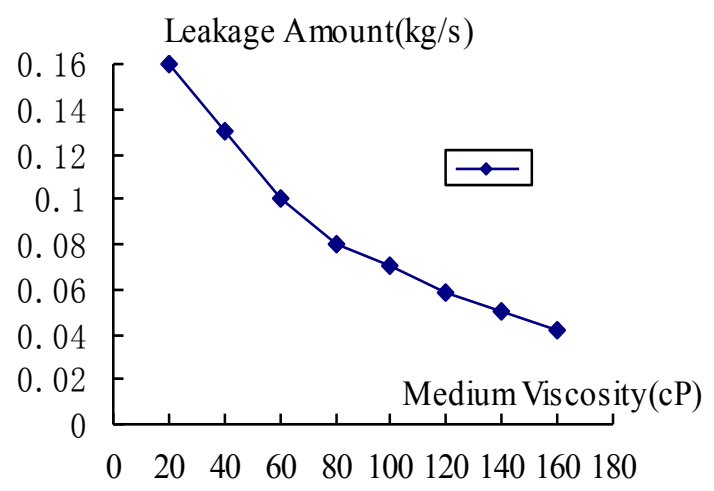

Fig. 8. Changes of Leakage Amount with Medium Viscosity 
The leakage amounts are $0.15 \mathrm{~kg} / \mathrm{s}$ and $0.04 \mathrm{~kg} / \mathrm{s}$ when the medium viscosities are $35 \mathrm{cP}$ and $160 \mathrm{cP}$, respectively, which indicate that the leakage amount decreases with the increase in medium viscosity. The curve has a large and small slope under low and high viscosities, respectively, which indicates that the leakage amount considerably decreases and then gradually stabilizes as the medium viscosity increases.

\subsection{Influence of Clearance on Leakage Amount}

The clearance between the sealing ring and the pump barrel, which serves as an important structural parameter of soft plunger pumps, has a direct influence on leakage amount. Figure 9 demonstrates the leakage amount under different clearances.

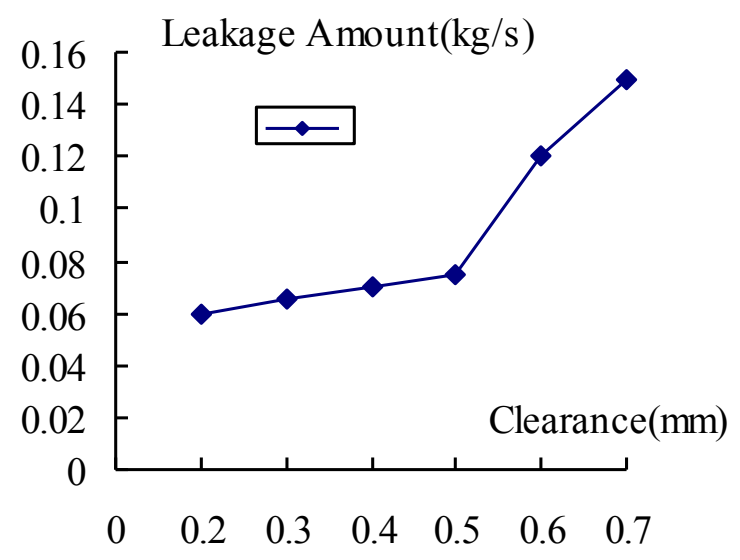

Fig. 9. Changes of Leakage Amount with Clearance

The clearances were obtained by changing the grid models and calculating the control parameters. The proposed model demonstrated a large range of clearance. To facilitate the study, the clearances were set to $0.2,0.3,0.4,0.5,0.6$, and $0.7 \mathrm{~mm}$, which corresponded to the leakage amounts of $0.06,0.065,0.07,0.075,0.12$, and $0.16 \mathrm{~kg} / \mathrm{s}$, respectively. When the clearance is larger than $0.5 \mathrm{~mm}$, the leakage amount increases considerably with the increase in clearance. The clearance should be properly controlled to maintain the leakage amount within an ideal range.

\subsection{Influence of Velocity on Leakage Amount}

The volumetric efficiency was calculated under different clearances $(0.5 \mathrm{~mm}$ and $1 \mathrm{~mm})$ to investigate the leakage amount under different velocities, as shown in Figure 10. From the figure, the volumetric efficiencies are $0.35,0.55$, $0.66,0.7$, and 0.72 when the velocities are 50,100,150,200, and $250 \mathrm{~L} / \mathrm{min}$, respectively. The volumetric efficiency increases considerably with the increase in velocity and then remains constant at 0.7 after the velocity reaches $150 \mathrm{~L} / \mathrm{min}$.

The relationship between the volumetric efficiency and the leakage amount can be expressed as follows:

$$
\eta=1-\frac{q}{Q}
$$

where $q$ is the leakage amount of soft plunger pumps, and $Q$ is the theoretical value of clearance flows.

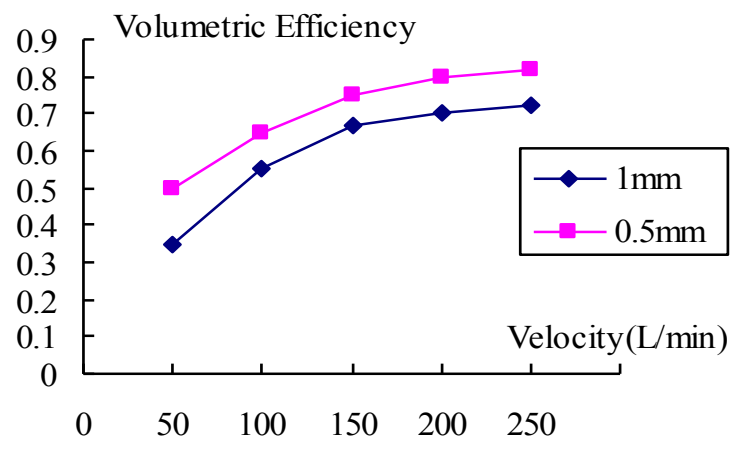

Fig. 10. Changes of Volumetric Efficiency with Velocity

\section{Conclusion}

A physical model for soft plunger pumps was constructed to reveal the relationship between the leakage amount and various influence factors, namely, pressure difference, velocity, medium viscosity, and clearance. The leakage amount of soft plunger pumps was analyzed on the basis of the combination of numerical simulation and experimental investigation. The conclusions could be drawn as follows:

(1) The leakage amount is positively correlated with pressure difference. After the outlet and inlet static pressure difference is determined, the pressure difference can be reasonably distributed by determining the number of stages. The number of stages of soft plunger pumps is reasonably selected under the premise of satisfying the pumping efficiency requirements to reduce material waste and save costs.

(2) The leakage amount is negatively correlated with medium viscosity. The leakage amount decreases with the increase in medium viscosity, which proves the adaptability of soft plunger pumps to high-viscosity fluids.

(3) The leakage amount is positively correlated with clearance. The leakage amount increases with the clearance, especially when the clearance is larger than $0.5 \mathrm{~mm}$. In view of the stresses of PEEK and the actual conditions, the clearance cannot be excessively small. Therefore, the clearance is eventually maintained in the range of $0.2-0.5$ $\mathrm{mm}$.

(4) The leakage amount is negatively correlated with velocity. Under low velocities, the leakage amount decreases while the volumetric efficiency increases with the velocity. After the velocity reaches a certain value, the leakage amount and the volumetric efficiency gradually stabilize.

This study combines laboratory experiment with theoretical investigation to reveal the influences of the operational and structural parameters of soft plunger pumps on the leakage amount. The proposed model is simple and practical and has certain reference value for subsequent development of soft plunger pumps. However, given the lack of on-site monitoring data, further studies should be conducted to combine the monitoring data with the proposed model to understand well the operation rules of soft plunger pumps under complex conditions.

This is an Open Access article distributed under the terms of the Creative Commons Attribution License 


\section{References}

1. Rahul Dinesh Gala, Kuhu Priyamvada, "Preventing Gas Locking in Sucker Rod Pumps Using an Actuated Traveling Solenoid Valve". Society of Petroleum Engineers 165039, 2012, pp.98-102.

2. Zakharov, B S, "Special Types of Sucker-Rod Pumps". Chemical and Petroleum Engineering, 44(3), 2008, pp.217-223.

3. Abdus Samad, Swapan Paruya, Samarjit Kar, Suchismita Roy, "Gas Interference in Sucker Rod Pump". In: American Institute of Physics

Conference Proceedings. American Institute of Physics, USA: AIP, 2010, pp.274-281.

4. Wang Yanli, Ran Lingguo, Guo Qun, Zhang Yingshan, "Research and Application of Flexible Plunger \& Retrievable Standing Valve Pumps”. Oil Drilling \& Production Technology, 32(2), 2010, pp.68-70.

5. Cui Lifeng, Chu Guiliang, Zhang Chundong, "Application of Soft Plunger Pump with Hydraulic Start-up in Sand Producing Block". Special Oil \& Gas Reservoirs, 9(5), 2002, pp. 77-79.

6. Wang Yan, "Research on Key Technologies of Stuck Pump in ASP Flooding”. Northeast Petroleum University, 2014, pp. 37-38.

7. Sun Lei, Sun Xihuan, Li Yongye, Jing Yuanhao, "Cyclical Slit Flow of Concentricity under Different Diameter Ratios". Yellow River, 36(11), 2014, pp. 110-112.

8. Yan You, Li Jingbo, Qiao Hongfeng, "A Brief Analysis of the Improvement of the Plunger Processing Technology". Science and Technology and Enterprise, 2(30) ,2013, pp. 298-299.

9. Li Qiang, Sun Chunlong, Mo Fei, "Research and Application of the New Self-sealing Soft Plunger Pump”.Chemical Machinery, 09(6) ,2009, pp. 37-38.

10. Li Qiang, "Design and Experimental Research of Pump with Sectionalized Soft Plunger Used in ASP Flooding”. Oil Field Equipment, 43(4) ,2014, pp. 68-70.

11. Tripp H.A, "A Review Analyzing Beam-Pumped Wells". SPE 19031
12. Zhu WB, Zhang ZW, "Analysis of lubrication state of plunger seal friction pair in plunger pumps". J Sichuan Univ Sci Technol, 03(2),2009, pp. 28-30.

13. Charles H. Newton, Masud Behnia, "Numerical calculation of turbulent stratified gas-liquid pipe flows". International Journal of Multiphase Flow, 12(26), 2000, pp. 327-337.

14. Randle, Seth J, "Low Cost Rod Pumping Management in Kern River Field". Society of Petroleum Engineers 37501, pp.49-53.

15. Chi M, "Analysis and countermeasures of high-pressure plunger injection pump seal”. Pump Technology, 11(05), 2003, pp. 38-40.

16. Lu Yaojun, Zhou Lixing, Shen Xiong, "Application and comparison of different turbulence models in flow field calculation of liquidliquid cyclone separation pipe".Journal of tsinghua university (natural science edition), 41(2), 2001, pp. 105-106.

17. V.I.Metelkin, V.A. Krasnik, A.V. Savitskii, "Selection of promising materials for operation in friction assem-blies of oil-well pumps in crude oil medium". Problems of Wear Resistance and Reliability of Machines, 9(02), 1992, pp. 67-70.

18. Li Junliang, "Study on Flexible Plunger Pumping System". Doctoral Dissertation of China Petroleum University, China, 2010, pp.25-29.

19. Liu Xinfu, Qi Yaoguang, Liu Chunhua, "Prediction of Flowing Bottomhole Pressures for Two-Phase Coalbed Methane Wells". Acta Petrolei Sinica, 31(6) , 2010, pp.998-1003.

20. CA Connally, CR Sandberg, N Stein, "Volumetric Efficiency of Sucker Rod Pumps When Pumping Gas-Oil Mixtures". Journal of Petroleum Technology, 5(10) , 2013, pp.265-270.

21. Wang Feng, Zhang Yingan, Pan Ruosheng, Yin Guojun, Ma Dongmei, SAN Antonio, "CO2 Flooding Lifting Technology in Oil Well with High Gas-Oil Ratio in Low Permeability Oilfield". In: SPE Asia Pacific Oil and Gas Conference and Exhibition, State of Texas, USA: SPE, 2012, pp.205-209. 